\title{
Long-Term Safety and Effectiveness of Linagliptin in Japanese Patients with Type 2 Diabetes Mellitus: A 3-Year Post-Marketing Surveillance Study
}

Fumiko Yamamoto · Yuriko Unno · Tomoo Okamura •

Rie Ikeda $\cdot$ Kaori Ochiai $\cdot$ Naoyuki Hayashi

Received: August 28, 2019 / Published online: November 11, 2019

(C) The Author(s) 2019

\section{ABSTRACT}

Introduction: Clinical trials of linagliptin in Japanese patients conducted to date have had limited observational periods; therefore, there is a need for additional longer-term real-world data. The aim of this study was to investigate the long-term safety and effectiveness of linagliptin in routine clinical practice.

Methods: This was a prospective, observational, post-marketing surveillance study conducted over 156 weeks in patients with type 2 diabetes mellitus who started linagliptin monotherapy. The primary endpoint was the incidence of adverse drug reactions (ADRs). The secondary endpoint was the change in glycated hemoglobin (HbA1c) from baseline to last available

Enhanced Digital Features To view enhanced digital features for this article go to: https://doi.org/10.6084/ m9.figshare.10068269.

Electronic supplementary material The online version of this article (https://doi.org/10.1007/s13300019-00723-x) contains supplementary material, which is available to authorized users.

F. Yamamoto $(\bowtie) \cdot$ Y. Unno $\cdot$ T. Okamura .

R. Ikeda $\cdot$ N. Hayashi

Medicine Division, Nippon Boehringer Ingelheim

Co., Ltd., Tokyo, Japan

e-mail: fumiko.yamamoto@boehringer-

ingelheim.com

K. Ochiai

PMS Division, EPS Corporation, Tokyo, Japan observation. Other effectiveness endpoints included the change in HbA1c and change in fasting plasma glucose (FPG) from baseline to week 26 and over the course of the treatment period.

Results: Overall, 2235 and 2054 patients were included in the safety and effectiveness analysis sets, respectively. Patients were mostly male $(58.4 \%)$, and the mean age was 66.7 years. The incidence of ADRs was $10.7 \%(n=240)$. The most frequent ADRs according to MedDRA preferred terms were diabetes mellitus $(n=35$ patients, $1.6 \%)$, constipation $(n=21,0.9 \%)$, diabetes mellitus inadequate control $(n=13$, $0.6 \%)$ and hypertension $(n=13,0.6 \%)$. The mean change in $\mathrm{HbA} 1 \mathrm{c}$ from baseline to last observation was $-0.67 \%$ [standard deviation (SD) $1.27 \%$, 95\% confidence interval -0.72 , - 0.61]. At week 26, HbA1c and FPG showed mean $\pm \mathrm{SD}$ changes from baseline of $-0.73 \pm 1.20 \%$ and $-21.02 \pm 44.33 \mathrm{mg} / \mathrm{dL}$, respectively, that were sustained until week 156 . Conclusions: In Japanese patients with type 2 diabetes mellitus, linagliptin produced sustained reductions in HbA1c and had a safety profile consistent with the established safety profile of linagliptin.

Trial Registration: ClinicalTrials.gov (NCT016 50259).

Keywords: DPP-4 inhibitor; Japanese; Linagliptin; Long-term; Post-marketing surveillance; Type 2 diabetes mellitus 


\section{Key Summary Points}

Why carry Out This Study?

The study was performed to meet regulatory obligations. The Japanese Pharmaceutical Affairs Law requires manufacturers of pharmaceutical agents to conduct a post-marketing surveillance study in order to accumulate safety and efficacy data on the product in the context of routine clinical practice.

This paper presents the final results of a 3 -year post-marketing surveillance study of linagliptin based on data for up to 156 weeks.

\section{What was Learned from the Study?}

The safety profile of linagliptin shown in this study is consistent with the established safety profile of linagliptin.

Linagliptin provided sustained reductions from baseline in glycated hemoglobin (mean \pm standard deviation $[\mathrm{SD}]$ change to last observation $-0.67 \pm 1.27 \%)$ and fasting plasma glucose (mean \pm SD change to week $156-20.41 \pm 43.79 \mathrm{mg}$ / $\mathrm{dL}$ ).

These findings confirm the safety and effectiveness of long-term linagliptin monotherapy in Japanese patients with type 2 diabetes mellitus in routine clinical practice.

\section{INTRODUCTION}

The prevalence of type 2 diabetes mellitus (T2DM) in Japan is increasing, and the number of elderly patients with T2DM is also expected to increase as a result of the aging Japanese population [1].
The Japan Diabetes Society recommends glucose-lowering drugs for patients with T2DM who do not achieve glycemic control with medical nutrition therapy and/or increased physical activity [2]. Oral glucose-lowering drugs currently available for the treatment of T2DM include sulfonylureas, biguanides, alphaglucosidase inhibitors, thiazolidinediones, glinides, dipeptidyl peptidase-4 (DPP-4) inhibitors and sodium-glucose transport protein 2 inhibitors [2]. Guidelines recommend that the choice of glucose-lowering drug should be based on the patient's individual circumstances and the efficacy and tolerability of the drug [2].

Linagliptin is a DPP-4 inhibitor that is used in the treatment of T2DM [3]. It acts by increasing the levels of the active incretin hormones glucagon-like peptide 1 and glucose-dependent insulinotropic polypeptide, both of which are normally inactivated by DPP-4, which in turn increases the synthesis and secretion of insulin in a glucose-dependent manner and reduces hepatic gluconeogenesis [4]. Linagliptin (Trazenta ${ }^{\circledR}$; Boehringer Ingelheim, Ingelheim, Germany) was approved for the treatment of patients with T2DM in Japan in 2011.

In phase III clinical trials of linagliptin in Caucasian and Japanese patients with T2DM, the proportion of patients who achieved glycated hemoglobin (HbA1c) levels of $<7.0 \%$ was significantly higher in patients who received linagliptin monotherapy than in those who received placebo [5-7]. Linagliptin was well tolerated by the patients participating in these studies, with few instances of hypoglycemia [5-8]. The efficacy of linagliptin has also been confirmed in elderly patients with T2DM [9].

Previous clinical trials of linagliptin, especially those conducted in Japanese patients, had several limitations, such as use of concomitant medications, short observation periods, low number of elderly patients and exclusion of patients with complications. The aim of the post-marketing surveillance study reported here was to investigate the long-term safety and effectiveness of linagliptin in Japanese patients with T2DM in routine clinical practice. An interim analysis that included effectiveness data up to 78 weeks has been published previously 
[10]. In this article, we present the final results of this post-marketing surveillance study based on data for up to 156 weeks, along with subgroup analysis by age on the safety and effectiveness of linagliptin and an analysis of the effectiveness of linagliptin according to body mass index (BMI).

\section{METHODS}

\section{Study Design, Patients and Data Collection}

This was a prospective, observational, postmarketing surveillance study conducted between July 2012 and October 2017 at 596 sites in Japan (ClinicalTrials.gov identifier: NCT01650259). The study was performed to meet regulatory obligations. The Japanese Pharmaceutical Affairs Law requires manufacturers of pharmaceutical agents to conduct a post-marketing surveillance study in order to accumulate safety and efficacy data on the product in the context of routine clinical practice. Linagliptin was first launched as initial monotherapy in Japan on 1 July 2011, and the indication was expanded to include add-on therapy to oral antidiabetic drugs and/or insulin on 25 March 2013.

Linagliptin-naïve patients with T2DM who were initiating treatment with linagliptin $(5 \mathrm{mg}$ once daily) monotherapy, according to the original (2011) Japanese package insert, were enrolled in the study between July 2012 and July 2014. Patients were followed up for 156 weeks or until linagliptin therapy was discontinued. The use of additional glucose-lowering drugs was permitted after enrollment because the survey reflected routine clinical use of linagliptin. All treatment decisions were made at the attending physician's discretion. This approach is fully compliant with Japanese Good Post-marketing Study Practice (GPSP) regulations. The protocol for this post-marketing surveillance was approved by the Ministry of Health, Labour and Welfare of the Japanese Government. This study involved the collection of anonymous data from clinical settings and, therefore, it was not necessary to obtain informed consent from patients.

This study collected data on the baseline characteristics of patients and on $\mathrm{HbA} 1 \mathrm{c}$, fasting plasma glucose (FPG), body weight, estimated glomerular filtration rate, vital signs, electrocardiograms, laboratory tests and adverse events (AEs) at, or close to, the following time points: $12,26,40,52,64,78$, 104,130 and 156 weeks after the start of treatment, or at discontinuation. Since patient visits to their treating physician were according to their routine care, data were collected at the visit closest to these time points; consequently, listed time points during the observation period are approximate. The laboratory analyses were conducted using routine clinical assays at the institutions participating in the study.

Data were collected using the electronic case report forms (eCRF) and entered into the electronic data capture (EDC) system. Separate eCRFs were completed for each patient at 26, 78 and 156 weeks after the start of treatment, or at discontinuation, and each CRF was transmitted to the EDC system upon completion. AE data could be sent to the EDC system at any time.

\section{Endpoints}

The primary endpoint of this study was the incidence of adverse drug reactions (ADRs), defined as AEs for which the causal relationship with linagliptin was assessed as definite or probable, or for which the causal relationship with linagliptin could not be excluded by either the investigator or sponsor (or both). AEs were coded using the Medical Dictionary for Regulatory Activities (MedDRA) version 20.1 (https:// www.meddra.org/how-to-use/support-docume ntation/english. The protocol specified that "exacerbation of the underlying disease" or "worsening of complications" should be collected as AEs in this survey. When physicians reported worsening control of diabetes in patients' eCRFs, the MedDRA preferred term (PT) category of "Diabetes mellitus" and lowest level term (LLT) of 'diabetes mellitus 
aggravated' were applied. When physicians reported 'worsening control of blood sugar' or similar on a patient's eCRF, the MedDRA LLT of 'loss of control of blood sugar' or 'diabetes mellitus inadequate control' within the PT category of "Diabetes mellitus inadequate control" was applied.

The secondary endpoint was the change in HbA1c from baseline to the last available observation. Other effectiveness endpoints included: change in $\mathrm{HbA1c}$ from baseline to week 26; change in HbA1c over the course of the treatment period; change in FPG from baseline to week 26; and change in FPG over the course of the treatment period.

\section{Statistics}

The planned sample size was 1500 patients. This sample size was chosen because it would allow for the detection of ADRs with an incidence of $0.20 \%$ with $95 \%$ probability. Enrollment was conducted using the continuous investigation system, wherein all patients who fulfilled the inclusion criteria were enrolled until the planned patient number for each facility (5-10) was reached.

Safety was evaluated in the safety analysis set, which included all patients who received linagliptin as monotherapy with the exception of those who had no data after enrollment or those with invalid registration. Effectiveness was evaluated in the effectiveness analysis set, which included all patients in the safety analysis set with the exception of those who had no effectiveness data and/or did not have T2DM.

As the analyses in this study were exploratory and descriptive, no statistical tests were performed except for the odds ratio (OR) and 95\% confidence intervals (CI) for ADRs in patients aged $\geq 65$ versus $<65$ years. Baseline, safety and effectiveness data were summarized using descriptive statistics. For continuous variables, mean and standard deviation (SD) and median, minimum, maximum and $95 \% \mathrm{CI}$ were calculated, while frequencies and proportions were calculated for categorical variables. Mixed model for repeated measures (MMRM) analyses were performed for change in HbA1c and FPG over the course of the observational period. Subgroup analyses were performed by baseline age $(<65$ and $\geq 65$ years) for the primary and secondary endpoints, and by baseline BMI $\left(<25\right.$ and $\left.\geq 25 \mathrm{~kg} / \mathrm{m}^{2}\right)$ for the secondary endpoints. The incidence of hypoglycemia was also analyzed according to baseline age $(<65$, $\geq 65$ to $<75$ and $\geq 75$ years). In addition, HbA1c over time was assessed in the subgroup of patients who received linagliptin as monotherapy with no additional glucose-lowering drugs overall and by baseline age $(<65$ and $\geq 65$ years $)$ and by baseline BMI $(<25$ and $\geq 25 \mathrm{~kg} / \mathrm{m}^{2}$ ). All statistical analyses were performed using SAS version 9.4 (SAS Institute, Cary, NC, USA).

\section{RESULTS}

Overall, 2235 patients were included in the safety analysis set and 2054 patients were included in the effectiveness analysis set (Fig. 1).

For patients included in the safety analysis set, the mean \pm SD duration of treatment with linagliptin was $115.1 \pm 58.8$ weeks. Over $50 \%$ of patients received linagliptin for $\geq 144$ weeks. Duration of exposure was between 144 and 156 weeks in $447(20.0 \%)$ patients and 156 weeks in 884 (39.6\%) patients.

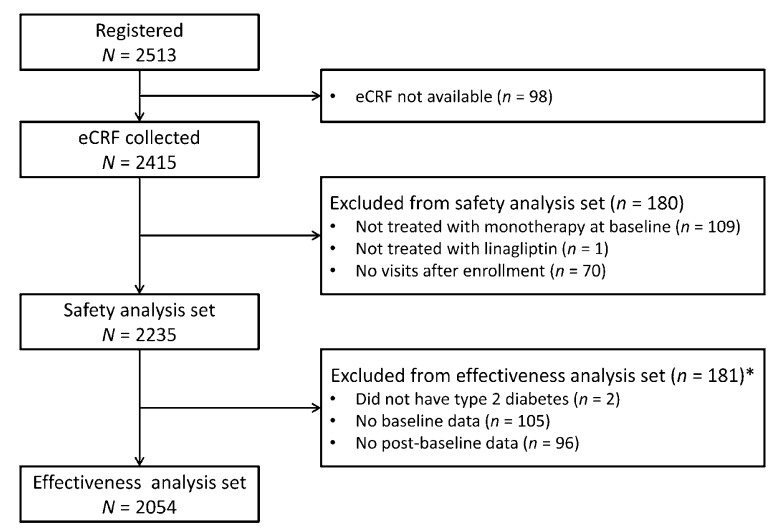

Fig. 1 Flow chart of patient inclusion. Asterisk refers to exclusion criteria: patients could be excluded for multiple reasons. eCRF Electronic case report form 
Patients were mostly male $(58.4 \%)$, with a mean age of $66.7 \pm 12.5$ years and a mean BMI of $25.2 \pm 4.2 \mathrm{~kg} / \mathrm{m}^{2}$ (Table 1 ). The majority of patients $(91.1 \%)$ had not previously received glucose-lowering drugs.

\section{Safety}

The incidence of ADRs was 10.7\% $(n=240)$ in the safety analysis set (Table 2 ). The most frequently reported ADRs using MedDRA PTs were diabetes mellitus $(n=35,1.6 \%)$, constipation $(n=21,0.9 \%)$, diabetes mellitus inadequate control $(n=13,0.6 \%)$ and hypertension $(n=13,0.6 \%$; Table 2$)$. Of the 48 patients with worsening diabetes (defined by the MedDRA PT "diabetes mellitus" or "diabetes mellitus inadequate control"), 42 were receiving oral antidiabetic medications in addition to linagliptin. Linagliptin was discontinued in 18 of the 48 patients who experienced worsening diabetes; the remaining 30 patients with these events completed the study. The reasons for discontinuation among these 18 patients were AEs $(n=8)$, insufficient efficacy $(n=6)$, improvement $(n=1)$, other reason $(n=1)$ or lost to follow-up/unknown $(n=2)$.

The incidence of ADRs did not continuously increase during the observational period. The incidence of ADRs leading to discontinuation was $3.3 \%(n=74)$.

Serious ADRs were reported in 35 patients $(1.6 \%)$ in the safety analysis set. The most frequently reported serious ADR was cerebral infarction $(n=4,0.2 \%$; Table 2$)$. The incidence rates of ADRs of special interest [hypoglycemia, pancreatitis, hypersensitivity (angioedema, urticaria, rash, bronchoconstriction), intestinal obstruction, hepatic dysfunction, skin lesions, pemphigoid, infections, worsening of renal function, pancreatic cancer, cardiac failure and interstitial lung disease] were low (Table 3). No drug-related pancreatitis or pemphigoid was reported in this study.

The incidence of ADRs was higher in patients aged $\geq 65$ years $(n=160 / 1333$ patients, $12.0 \%)$ than in those aged $<65$ years $(n=80 / 902,8.9 \%$; OR $1.4 ; 95 \%$ CI 1.06-1.86). In patients aged $\geq 65$ years, the most frequently reported ADRs
Table 1 Baseline characteristics of patients who received linagliptin $5 \mathrm{mg}$ once daily

\begin{tabular}{|c|c|}
\hline Characteristics & $\begin{array}{l}\text { Values }(N=2235 \\
\text { patients) }\end{array}$ \\
\hline \multicolumn{2}{|l|}{ Sex, $n(\%)$} \\
\hline Male & $1305(58.4)$ \\
\hline Female & $930(41.6)$ \\
\hline \multicolumn{2}{|l|}{ Age (years) } \\
\hline Mean $\pm S D$ & $66.7 \pm 12.5$ \\
\hline \multicolumn{2}{|c|}{ Age categories $[n(\%)]$} \\
\hline$<65$ years & $902(40.4)$ \\
\hline$\geq 65$ years & $1333(59.6)$ \\
\hline $65-74$ years & $678(30.3)$ \\
\hline$\geq 75$ years & $655(29.3)$ \\
\hline \multicolumn{2}{|l|}{ Body weight (kg) } \\
\hline$n$ & 1794 \\
\hline Mean $\pm S D$ & $64.5 \pm 14.0$ \\
\hline \multicolumn{2}{|l|}{ BMI $\left(\mathrm{kg} / \mathrm{m}^{2}\right)$} \\
\hline$n$ & 1643 \\
\hline Mean \pm SD & $25.2 \pm 4.2$ \\
\hline \multicolumn{2}{|c|}{ Duration of diabetes categories $[n(\%)]$} \\
\hline$\leq 1$ year & $501(22.4)$ \\
\hline$>1-5$ years & $461(20.6)$ \\
\hline$>5$ years & $1273(57.0)$ \\
\hline \multicolumn{2}{|c|}{ HbAlc (based on NGSP) (\%) } \\
\hline$n$ & 2130 \\
\hline Mean \pm SD & $7.4 \pm 1.4$ \\
\hline \multicolumn{2}{|l|}{$\mathrm{FPG}(\mathrm{mg} / \mathrm{dL})$} \\
\hline$n$ & 846 \\
\hline Mean \pm SD & $151.2 \pm 50.8$ \\
\hline \multicolumn{2}{|c|}{ Concomitant diagnosis $[n(\%)]$} \\
\hline No & $432(19.3)$ \\
\hline Yes $^{a}$ & $1764(78.9)$ \\
\hline Hypertension & $1234(55.2)$ \\
\hline Dyslipidemia & $498(22.3)$ \\
\hline
\end{tabular}


Table 1 continued

\begin{tabular}{|c|c|}
\hline Characteristics & $\begin{array}{l}\text { Values }(N=2235 \\
\text { patients) }\end{array}$ \\
\hline Hyperlipidemia & $353(15.8)$ \\
\hline Hypercholesterolemia & $198(8.9)$ \\
\hline Hyperuricemia & $197(8.8)$ \\
\hline Hepatobiliary disorder ${ }^{\mathrm{b}}$ & $179(8.0)$ \\
\hline Chronic kidney disease & $101(4.5)$ \\
\hline Unknown & $39(1.7)$ \\
\hline \multicolumn{2}{|c|}{ Complications of diabetes $[n(\%)]$} \\
\hline Diabetic nephropathy & $60(2.7)$ \\
\hline Diabetic neuropathy & $13(0.6)$ \\
\hline Diabetic retinopathy & $14(0.6)$ \\
\hline \multicolumn{2}{|c|}{ Cardiovascular history $[n(\%)]$} \\
\hline No & $1872(83.8)$ \\
\hline Yes & $324(14.5)$ \\
\hline Unknown & $39(1.7)$ \\
\hline \multicolumn{2}{|c|}{ Prior antidiabetic medication $[n(\%)]$} \\
\hline No & $2036(91.1)$ \\
\hline Yes & $199(8.9)$ \\
\hline 1 drug & $171(7.7)$ \\
\hline$\geq 2$ drugs & $28(1.3)$ \\
\hline \multicolumn{2}{|c|}{ Duration of linagliptin treatment (weeks) } \\
\hline$n$ & 2235 \\
\hline $\begin{array}{l}\text { Median }[\text { minimum, } \\
\text { maximum] }\end{array}$ & $154.1[0.6,241.0]$ \\
\hline
\end{tabular}

$B M I$ Body mass index, FPG Fasting plasma glucose, $H b A 1 c$ Glycated hemoglobin, NGSP National Glycohemoglobin Standardization Program, SD Standard deviation

${ }^{a}$ Main complications are shown

${ }^{b}$ Defined as one of the following standardized Medical Dictionary for Regulatory Activities (MedDRA) queries (SMQs): (1) hepatic disorders (narrow) (SMQ 20000005); (2) biliary disorders (narrow) (SMQ 20000118)

were constipation $(n=18,1.4 \%)$, worsening of diabetes mellitus ( $n=16,1.2 \%)$, hypertension $(n=10,0.8 \%)$ and dizziness $(n=8,0.6 \%)$. In
Table 2 Incidence of adverse drug reactions and serious adverse drug reactions

\begin{tabular}{|c|c|}
\hline $\operatorname{ADRs}[n(\%)]$ & $\begin{array}{l}\text { Incidence }(N=2235 \\
\text { patients) }\end{array}$ \\
\hline Any ADRs & $240(10.7)$ \\
\hline \multicolumn{2}{|l|}{ ADRs occurring in $\geq 5$ patients } \\
\hline Diabetes mellitus & $35(1.6)$ \\
\hline Constipation & $21(0.9)$ \\
\hline $\begin{array}{l}\text { Diabetes mellitus inadequate } \\
\text { control }\end{array}$ & $13(0.6)$ \\
\hline Hypertension & $13(0.6)$ \\
\hline Hyperuricemia & $11(0.5)$ \\
\hline Hepatic function abnormal & $11(0.5)$ \\
\hline Dizziness & $9(0.4)$ \\
\hline Decreased appetite & $6(0.3)$ \\
\hline Weight increased & $6(0.3)$ \\
\hline $\begin{array}{l}\text { Gastroesophageal reflux } \\
\text { disease }\end{array}$ & $5(0.2)$ \\
\hline $\begin{array}{l}\text { ADRs leading to } \\
\text { discontinuation }\end{array}$ & $74(3.3)$ \\
\hline Serious ADRs & $35(1.6)$ \\
\hline \multicolumn{2}{|c|}{ Serious ADRs occurring in $\geq 2$ patients } \\
\hline Cerebral infarction & $4(0.2)$ \\
\hline Myocardial infarction & $3(0.1)$ \\
\hline Pancreatic carcinoma & $3(0.1)$ \\
\hline Death & $3(0.1)$ \\
\hline Sudden death & $2(0.1)$ \\
\hline Fall & $2(0.1)$ \\
\hline
\end{tabular}

$A D R$ Adverse drug reaction

patients aged $\geq 75$ years $(n=655)$, the most frequently reported ADRs were constipation $(n=10$, $1.5 \%)$, worsening of diabetes mellitus $(n=6$, $0.9 \%)$, decreased appetite $(n=5,0.8 \%)$, hypertension $(n=5,0.8 \%)$ and dizziness $(n=4,0.6 \%)$. In this latter age cohort, the incidence of constipation and dizziness was numerically $\geq 4$.0-fold higher $(n=10,1.5 \%$ and $n=4,0.6 \%$, respectively) than in patients aged $<65$ years $(n=3$, 
Table 3 Incidence of adverse drug reactions of special interest

\begin{tabular}{ll}
\hline $\begin{array}{l}\text { ADRs of special interest } \\
{[\boldsymbol{n}(\%)]}\end{array}$ & $\begin{array}{l}\text { Incidence }(\boldsymbol{N}=\mathbf{2 2 3 5} \\
\text { patients })\end{array}$ \\
\hline Hepatic dysfunction $^{\mathrm{a}}$ & $17(0.8)$ \\
Hypersensitivity & $14(0.6)$ \\
Worsening of renal function & $5(0.2)$ \\
Hypoglycemia $^{\mathrm{a}}$ & $3(0.1)$ \\
Intestinal obstruction $^{\mathrm{a}}$ & $3(0.1)$ \\
Pancreatic cancer & $3(0.1)$ \\
Cardiac failure $_{\text {Skin lesions }}$ & $3(0.1)$ \\
Infections $^{\text {Interstitial lung disease }}{ }^{\mathrm{a}}$ & $2(0.09)$ \\
Pancreatitis $^{\mathrm{a}}$ & $2(0.09)$ \\
Pemphigoid $^{\mathrm{a}}$ & 0 \\
\hline
\end{tabular}

$A D R$ Adverse drug reaction, MedDRA Medical dictionary for regulatory activities, $P T$ Preferred term, $S M Q$ Standardized MedDRA query, SOC System organ class

a Described in 'Clinically Significant Adverse reactions' of the Japanese package insert. Hepatic dysfunction: liverrelated investigations, signs and symptoms (SMQ: 20000008), cholestasis and jaundice of hepatic origin (subSMQ: 20000009), hepatitis, non-infectious (sub-SMQ: 20000010) and hepatic failure, fibrosis and cirrhosis and other liver damage-related conditions (sub-SMQ: 20000013); hypersensitivity: hypersensitivity (SMQ: 20000214); worsening of renal function: acute renal failure (SMQ: 20000003); hypoglycemia: hypoglycaemia (SMQ: 20000226); cardiac failure: cardiac failure (SMQ: 20000004); skin lesions: severe cutaneous adverse reactions (SMQ: 20000020); infections: infections and infestations (SOC: 10021881) and at least 1 serious criterion fulfilled; interstitial lung disease: interstitial lung disease (SMQ: 20000042); pemphigoid: pemphigoid (PT); intestinal obstruction (PT); pancreatic cancer (PT); pancreatitis: PTs specified by Boehringer Ingelheim (Ingelheim am Rhein, Germany)

$0.3 \%$ and $n=1,0.1 \%$, respectively). Hypoglycemia was reported in three patients: one in the $<65$ year group $(n=1 / 902,0.1 \%)$, one in the $\geq 65$ to $<75$ years group $(n=1 / 678,0.2 \%)$ and one in the $\geq 75$ year group $(n=1 / 655,0.2 \%)$.

\section{Effectiveness}

\section{Glycated Hemoglobin}

In patients included in the effectiveness analysis set, the mean $\pm \mathrm{SD}$ HbA1c level was $7.43 \pm 1.36 \%$ at baseline and $6.76 \pm 0.97 \%$ at the last observation. The mean change in HbA1c from baseline to the last observation was $-0.67 \%$ (SD 1.27\%, 95\% CI - 0.72 to -0.61 ). A decrease in HbA1c was seen at week 12 (mean change from baseline $-0.56 \%$ ), and at week 26 HbA1c was $6.69 \pm 0.89 \%$, indicating a mean \pm SD change from baseline of $-0.73 \pm 1.20 \%$. This decrease in HbA1c from baseline was sustained until week 156 (mean change from baseline over the treatment period ranged from -0.56 to $-0.77 \%$; Table 4$)$. Results of the MMRM analysis of HbA1c over time are presented in Fig. 2 a.

In patients aged $<65$ years, the mean \pm SD HbA1c at baseline and at last observation was $7.85 \pm 1.58$ and $6.94 \pm 1.12 \%$, respectively, and the mean change from baseline to the last observation was - $0.91 \%$ (SD 1.48, 95\% CI -1.01 to -0.80 ). In patients aged $\geq 65$ years, the mean \pm SD HbA1c at baseline and at last observation was $7.15 \pm 1.10$ and $6.64 \pm 0.83 \%$, respectively, and the mean change from baseline to the last observation was $-0.51 \%$ (SD $1.08,95 \%$ CI -0.57 to -0.45$)$. Results of the MMRM analysis of HbA1c over time by age are presented in Electronic Supplementary Material (ESM) Fig. 1. From week 26 until week 156 the decrease in HbA1c was sustained at between 6.79 and $6.88 \%$ in patients aged $<65$ years and at between 6.56 and $6.64 \%$ in patients aged $\geq 65$ years.

In patients with a BMI of $<25 \mathrm{~kg} / \mathrm{m}^{2}$, the mean \pm SD HbA1c at baseline and at last observation was $7.33 \pm 1.28$ and $6.70 \pm 0.88 \%$, respectively, and the mean change from baseline to the last observation was $-0.63 \%$ (SD $1.20,95 \% \mathrm{CI}-0.71$ to -0.55$)$. In patients with a BMI of $\geq 25 \mathrm{~kg} / \mathrm{m}^{2}$, the mean \pm SD HbA1c at baseline and at last observation was $7.59 \pm 1.49$ and $6.82 \pm 1.04 \%$, respectively; the mean change from baseline to the last observation was $-0.78 \%$ (SD $1.42,95 \%$ CI -0.88 to - 0.68). Results of the MMRM analysis of HbA1c over time by BMI are presented in ESM 
Table 4 Change in glycated hemoglobin and fasting plasma glucose from baseline

\begin{tabular}{llllll}
\hline HbAc1 and FPG & \multicolumn{5}{l}{ Change from baseline at } \\
\cline { 2 - 6 } & $\mathbf{1 2}$ weeks & $\mathbf{2 6}$ weeks & 52 weeks & 104 weeks & 156 weeks/EOT \\
\hline HbAlc (\%) & & 1725 & 1329 & 1169 & 1119 \\
$n$ & 1901 & $-0.73 \pm 1.20$ & $-0.70 \pm 1.18$ & $-0.76 \pm 1.25$ & $-0.69 \pm 1.20$ \\
Mean \pm SD & $-0.56 \pm 1.04$ & & & & \\
FPG (mg/dL) & & 475 & 289 & 256 & 245 \\
$n$ & 535 & $-21.02 \pm 44.33$ & $-18.26 \pm 39.57$ & $-19.39 \pm 44.73$ & $-20.41 \pm 43.79$ \\
\hline
\end{tabular}

EOT End of treatment

Fig. 2. There was a small decrease in body weight over the duration of linagliptin treatment, from a mean \pm SD of $64.52 \pm 13.97 \mathrm{~kg}$ at baseline to $63.74 \pm 13.88 \mathrm{~kg}$ at the end of the observation period (mean change of $-0.77 \mathrm{~kg}$, SD $4.43,95 \% \mathrm{CI}-1.00$ to -0.55 ).
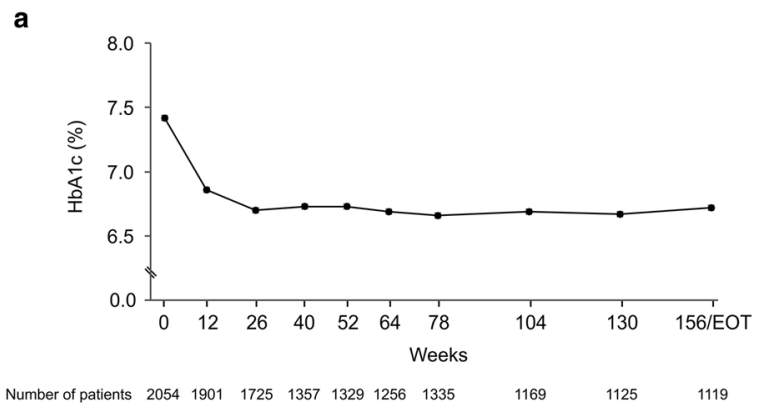

b

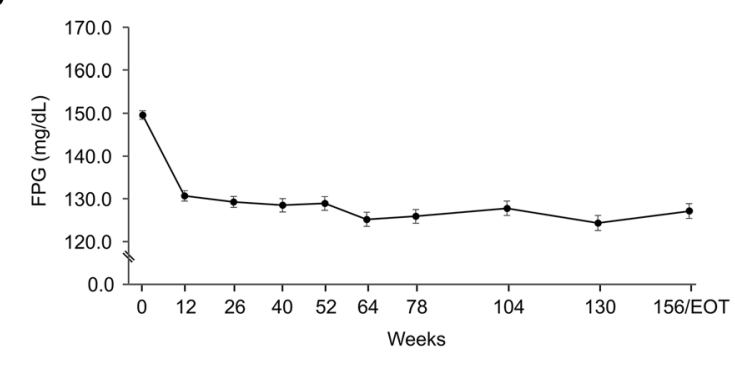

$\begin{array}{lllllllllll}\text { Number of patients } & 805 & 535 & 475 & 305 & 289 & 269 & 290 & 256 & 237 & 245\end{array}$

Fig. 2 Glycated hemoglobin ( $H b A 1 c ;$ a) and fasting plasma glucose $(F P G ; \mathbf{b})$ over time in patients who received linagliptin $5 \mathrm{mg}$. Data are shown as the adjusted mean \pm standard error. Mixed model for repeated measures analysis was performed. EOT End of treatment
During the study period, most of patients received linagliptin as monotherapy with no additional glucose-lowering drugs (82.3\%), while the proportions of the patients who received one and two or more additional glucose-lowering drugs were 12.4 and 5.3\%, respectively (ESM Fig. 3a). The median duration of linagliptin treatment in patients who received linagliptin monotherapy with no additional glucose-lowering drugs was 153.7 weeks, and the majority of patients received >144 weeks of treatment (ESM Fig. 3b).

In patients who received linagliptin monotherapy with no additional glucose-lowering drugs, the mean \pm SD HbA1c level was $7.28 \pm 1.25 \%$ at baseline and $6.67 \pm 0.93 \%$ at the last observation; the mean change in HbA1c from baseline to the last observation was $-0.61 \%$ (SD $1.14 \%, 95 \%$ CI -0.66 to -0.55 ). Results of the MMRM analysis of HbA1c over time in all patients who received linagliptin monotherapy with no additional glucose-lowering drugs, of HbA1c over time by age and of HbA1c over time by BMI are presented in ESM Fig. 4.

\section{Fasting Plasma Glucose}

Baseline FPG data were available for 805 patients, and the mean \pm SD FPG level was $150.72 \pm 50.36 \mathrm{mg} / \mathrm{dL}$. At week 26, FPG was $131.36 \pm 36.65 \mathrm{mg} / \mathrm{dL}$, indicating in a change from baseline of $-21.02 \pm 44.33 \mathrm{mg} / \mathrm{dL}$. At week 156, the FPG was $125.33 \pm 30.81 \mathrm{mg} / \mathrm{dL}$, 
indicating in a mean change from baseline of $-20.41 \pm 43.79 \mathrm{mg} / \mathrm{dL}$. Compared with baseline, FPG was decreased at week 12 $(-19.20 \mathrm{mg} / \mathrm{dL})$, and this decrease was maintained until week 156 (mean change from baseline -18.26 to $-23.12 \mathrm{mg} / \mathrm{dL}$; Table 4 ). Results of the MMRM analysis of FPG over time are presented in Fig. $2 b$.

In patients aged $<65$ years, the mean $\pm S D$ FPG level at baseline and at last observation was $160.52 \pm 54.59$ and $\quad 133.27 \pm 41.61 \mathrm{mg} / \mathrm{dL}$, respectively; the mean change from baseline to the last observation was $-28.11 \mathrm{mg} / \mathrm{dL}$ (SD $49.96,95 \%$ CI -33.88 to -22.35 ). In patients aged $\geq 65$ years, the mean \pm SD FPG level at baseline and at last observation was $143.63 \pm 45.83$ and $124.87 \pm 29.29 \mathrm{mg} / \mathrm{dL}$, respectively; the mean change from baseline to the last observation was $-16.48 \mathrm{mg} / \mathrm{dL}$ (SD $44.11,95 \%$ CI -20.76 to -12.21 ). Results of the MMRM analysis FPG over time by age are presented in ESM Fig. 5.

\section{DISCUSSION}

To our knowledge, this is the first report to assess the long-term use of linagliptin in a realworld setting in Japanese patients. Results of this multicenter, prospective, observational post-marketing surveillance study demonstrated that linagliptin, administered as monotherapy for up to 3 years, was well tolerated and led to clinically meaningful reductions in HbA1c and FPG in Japanese patients with T2DM.

ADRs occurred in $10.7 \%$ of patients, with the most frequently reported ADR-excluding deterioration of the primary disease, i.e. diabetes-was constipation (0.9\%); this finding is consistent with those of a previous phase III clinical study in Japanese patients [8]. In that phase III trial, the incidence of ADRs was 10.2\% in Japanese patients who received linagliptin $5 \mathrm{mg}$ once daily for up to 52 weeks, and the most frequent ADR was constipation (1.9\%) [8]. The incidence of ADRs that were not related to the deterioration of diabetes was $8.9 \%$. The large portion of ADRs reported were related to worsening of diabetes mellitus (diabetes mellitus, diabetes mellitus inadequate control, glycosylated hemoglobin increased and blood glucose increased). The ADRs related to worsening of diabetes mellitus may mean that treated patients could in general not obtain the expected effect with linagliptin and that therefore these ADRs could be considered in the analysis of effectiveness as well as safety.

In this study, the incidence of ADRs was higher in patients aged $\geq 65$ years than in those aged $<65$ years. In patients aged $\geq 65$ years, the incidence of constipation and dizziness was $\geq$ 4 -fold higher than in patients aged $<65$ years. The results of the safety subgroup analyses of this study are comparable with those of a pooled analysis of 22 placebo-controlled trials in which the incidence of ADRs increased with age [11]. In this latter pooled analysis, the incidence of ADRs was similar in patients who received linagliptin and those who received placebo across age subgroups [11]. It is important to note that the proportion of elderly patients (aged $\geq 75$ years) in the present study was $29 \%$, which is higher than that in previous clinical trials (5.6 and 1.9\%) [11, 12].

Hypoglycemia is listed as a clinically significant adverse reaction in the precautions section of the linagliptin Japanese product package insert, and physicians are urged to exercise caution with this $\mathrm{AE}$ [13]. The incidence of hypoglycemia in this study was low, as it was only reported in three patients. Results from the analysis of three age subgroups of patients ( $<65$ years, $\geq 65$ to $<75$ years and $\geq 75$ years) showed no marked differences in the incidence of hypoglycemia.

The results of the present study confirm the effectiveness of linagliptin in reducing HbA1c and FPG levels in Japanese patients with T2DM in a real-world setting. The findings of this study are also in line with those of a pooled analysis of data on Asian patients from 11 shortterm randomized controlled studies (observational period was 24-52 weeks) [12]. In these studies, linagliptin was effective in patients regardless of age. The control of HbA1c with linagliptin in elderly patients with T2DM in the present study is consistent with that observed in a previous clinical trial [9]. In addition, the 
decrease in HbA1c observed in this study was seen regardless of baseline BMI.

Subgroup analyses conducted in the patients who received linagliptin as monotherapy with no additional glucose-lowering drugs showed a sustained decrease in $\mathrm{HbA1c}$ regardless of age or BMI at baseline.

This study had several limitations. Firstly, concomitant antidiabetic medications could be added and changes in diet and exercise therapy were permitted after the initiation of linagliptin therapy. Secondly, this study was not designed as a comparative study with regard to either effectiveness or safety. Thirdly, the results, including effectiveness, were summarized using descriptive statistics, and no statistical tests were conducted. Despite these limitations, this study demonstrates the safety and effectiveness of linagliptin administered as monotherapy in Japanese patients with T2DM in a real-world setting and provides clinically important information on the tolerability and clinical effectiveness of long-term use of linagliptin.

\section{CONCLUSIONS}

In conclusion, this report provides data on the safety and effectiveness of long-term use of linagliptin initiated as monotherapy for the treatment of T2DM in a real-world setting. The safety profile of linagliptin shown in this study is consistent with the established safety profile of linagliptin. Sustained decreases of HbA1c and FPG with linagliptin in Japanese patients with T2DM were demonstrated, regardless of age or $\mathrm{BMI}$ at baseline in a real-world setting.

\section{ACKNOWLEDGEMENTS}

The authors thank all of the participants in this study.

Funding. Nippon Boehringer Ingelheim Co., Ltd. and Eli Lilly Japan K.K. funded this study and the Rapid Service Fee.

Medical Writing Assistance. The authors thank Georgii Filatov of inScience
Communications, Springer Healthcare, for medical writing support in the preparation of this manuscript. Funding for medical writing assistance was provided by Nippon Boehringer Ingelheim Co., Ltd. and Eli Lilly Japan K.K.

Authorship. All named authors meet the International Committee of Medical Journal Editors (ICMJE) criteria for authorship for this article, take responsibility for the integrity of the work as a whole, and have given their approval for the final version to be published.

Authorship Contributions. Rie Ikeda and Kaori Ochiai made substantial contributions to conception and design; Rie Ikeda made substantial contributions to data acquisition and analysis; Kaori Ochiai provided statistical expertise; all authors provided contributions to data interpretation, drafting the manuscript, revising for important intellectual content and approval of the final version.

Prior Presentation. Results of this study have been presented in Poster III-P-179 at the 61st Annual Meeting of the Japan Diabetes Society that took place on May 24-26, 2018, in Tokyo, Japan.

Disclosures. Fumiko Yamamoto is an employee of Nippon Boehringer Ingelheim Co., Ltd. Tomoo Okamura is an employee of Nippon Boehringer Ingelheim Co., Ltd. Rie Ikeda is an employee of Nippon Boehringer Ingelheim Co., Ltd. Naoyuki Hayashi is an employee of Nippon Boehringer Ingelheim Co., Ltd. Kaori Ochiai is an employee of EPS Corporation. Yuriko Unno was an employee of Nippon Boehringer Ingelheim Co., Ltd. at the time the study was conducted but is now employed at the Department of Pharmacy, Shizuoka Rheumatism Orthopedics Rehabilitation Hospital, Shizuoka, Japan.

Compliance with Ethics Guidelines. This approach is fully compliant with Japanese Good Post-marketing Study Practice (GPSP) regulations. The protocol for this post-marketing surveillance was approved by the Ministry of Health, Labour and Welfare of the Japanese Government. This study involved the collection 
of anonymous data from clinical settings and, therefore, it was not necessary to obtain informed consent from patients. All medical institutions who agreed to provide this anonymized data signed a contract with Nippon Boehringer Ingelheim Co., Ltd. or Eli Lilly Japan K.K.

Data Availability. The data that support the findings of this study are available from the corresponding author, Fumiko Yamamoto, upon reasonable request.

Open Access. This article is distributed under the terms of the Creative Commons Attribution-NonCommercial 4.0 International License (http://creativecommons.org/licenses/ by-nc/4.0/), which permits any noncommercial use, distribution, and reproduction in any medium, provided you give appropriate credit to the original author(s) and the source, provide a link to the Creative Commons license, and indicate if changes were made.

\section{REFERENCES}

1. Charvat H, Goto A, Goto M, et al. Impact of population aging on trends in diabetes prevalence: a meta-regression analysis of 160,000 Japanese adults. J Diabetes Investig. 2015;6:533-42.

2. Haneda $M$, Noda $M$, Origasa $H$, et al. Japanese clinical practice guideline for diabetes 2016. J Diabetes Investig. 2018;9:657-97.

3. Deeks ED. Linagliptin: a review of its use in the management of type 2 diabetes mellitus. Drugs. 2012;72:1793-824.

4. McKeage K. Linagliptin: an update of its use in patients with type 2 diabetes mellitus. Drugs. 2014;74:1927-46.

5. Barnett AH, Patel S, Harper R, et al. Linagliptin monotherapy in type 2 diabetes patients for whom metformin is inappropriate: an 18-week randomized, double-blind, placebo-controlled phase III trial with a 34-week active-controlled extension. Diabetes Obes Metab. 2012;14:1145-54.

6. Del Prato S, Barnett AH, Huisman H, Neubacher D, Woerle HJ, Dugi KA. Effect of linagliptin monotherapy on glycaemic control and markers of beta-cell function in patients with inadequately controlled type 2 diabetes: a randomized controlled trial. Diabetes Obes Metab. 2011;13:258-67.

7. Kawamori R, Inagaki N, Araki E, et al. Linagliptin monotherapy provides superior glycaemic control versus placebo or voglibose with comparable safety in Japanese patients with type 2 diabetes: a randomized, placebo and active comparator-controlled, double-blind study. Diabetes Obes Metab. 2012;14:348-57.

8. Araki E, Kawamori R, Inagaki N, et al. Long-term safety of linagliptin monotherapy in Japanese patients with type 2 diabetes. Diabetes Obes Metab. 2013;15:364-71.

9. Barnett AH, Huisman H, Jones R, von Eynatten M, Patel S, Woerle HJ. Linagliptin for patients aged 70 years or older with type 2 diabetes inadequately controlled with common antidiabetes treatments: a randomised, double-blind, placebo-controlled trial. Lancet. 2013;382:1413-23.

10. Unno Y, Ochiai K, Ikeda R, Hayashi N. Long-term safety and efficacy of linagliptin (Trazenta ${ }^{\circledR}$ Tablets $5 \mathrm{mg}$ ), a DPP-4 inhibitor, in patients with type 2 diabetes mellitus-interim report from special surveillance in patients who started linagliptin treatment as monotherapy. J New Rem Clin. 2018;67:667-88. (in Japanese).

11. Lehrke M, Marx N, Patel S, et al. Safety and tolerability of linagliptin in patients with type 2 diabetes: a comprehensive pooled analysis of 22 placebocontrolled studies. Clin Ther. 2014;36:1130-46.

12. Ning G, Bandgar T, Hehnke U, Lee J, Chan JCN. Efficacy and safety of linagliptin in 2681 Asian patients stratified by age, obesity, and renal function: a pooled analysis of randomized clinical trials. Adv Ther. 2017;34:2150-62.

13. World Health Organization. World Health Organization model list of essential medicines, 21st list (2019). Geneva: World Health Organization; 2019. 